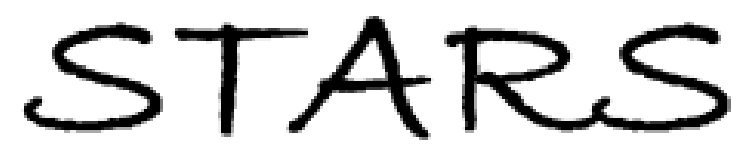

University of Central Florida

STARS

Faculty Bibliography 2000s

Faculty Bibliography

$1-1-2007$

\title{
Liquid mixing driven motions of floating macroscopic objects
}

Ming Su

University of Central Florida

Find similar works at: https://stars.library.ucf.edu/facultybib2000

University of Central Florida Libraries http://library.ucf.edu

This Article is brought to you for free and open access by the Faculty Bibliography at STARS. It has been accepted for inclusion in Faculty Bibliography 2000s by an authorized administrator of STARS. For more information, please contact STARS@ucf.edu.

\section{Recommended Citation}

Su, Ming, "Liquid mixing driven motions of floating macroscopic objects" (2007). Faculty Bibliography 2000s. 7689.

https://stars.library.ucf.edu/facultybib2000/7689

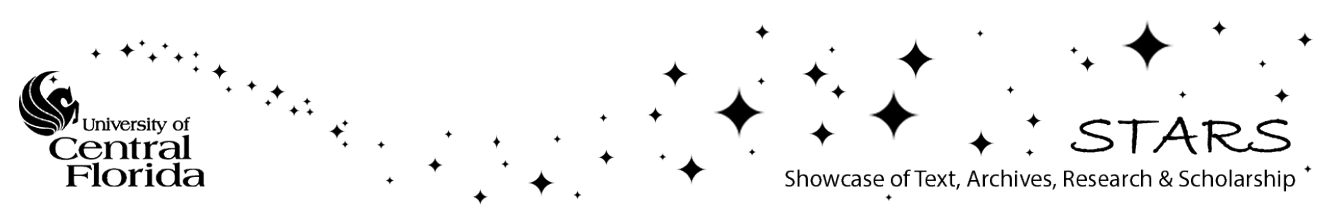




\section{Liquid mixing driven motions of floating macroscopic objects}

Cite as: Appl. Phys. Lett. 90, 144102 (2007); https://doi.org/10.1063/1.2719029

Submitted: 22 December 2006 . Accepted: 28 February 2007 . Published Online: 02 April 2007

\section{Ming Su}

\section{ARTICLES YOU MAY BE INTERESTED IN}

Marangoni spreading due to a localized alcohol supply on a thin water film

Physics of Fluids 27, 032003 (2015); https://doi.org/10.1063/1.4915283

Self-propelling rotator driven by soluto-capillary marangoni flows

Applied Physics Letters 110, 131604 (2017); https://doi.org/10.1063/1.4979590

The "Cheerios effect"

American Journal of Physics 73, 817 (2005); https://doi.org/10.1119/1.1898523

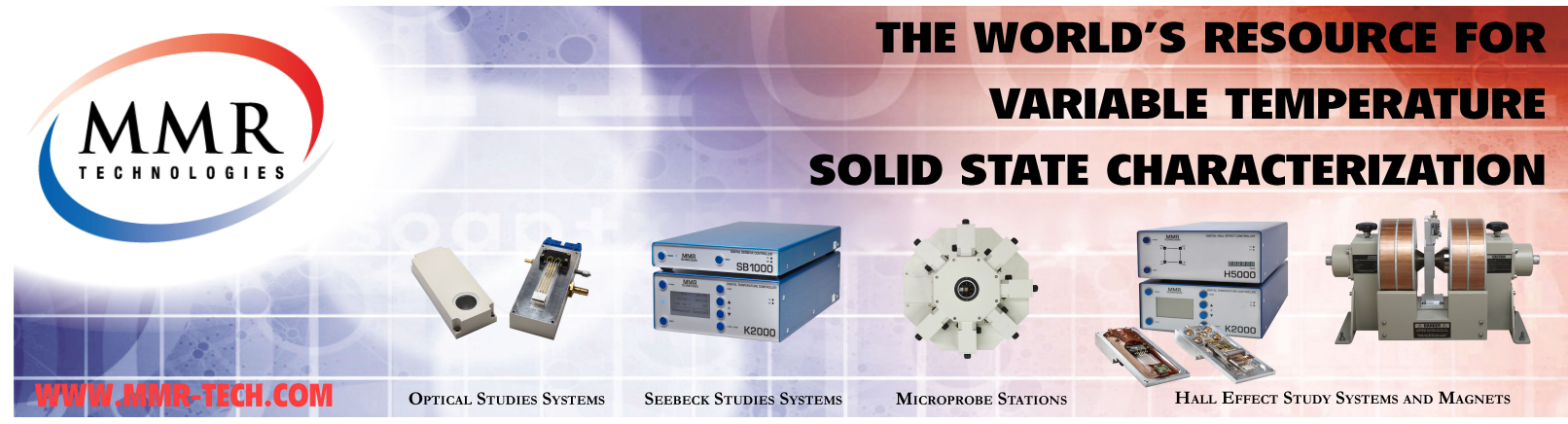




\title{
Liquid mixing driven motions of floating macroscopic objects
}

\author{
Ming Su ${ }^{\mathrm{a})}$ \\ NanoScience Technology Center, University of Central Florida, Orlando, Florida 32826; \\ Department of Mechanical, Materials, and Aerospace Engineering, University of Central Florida, Orlando, \\ Florida 32826; and Department of Chemistry, University of Central Florida, Orlando, Florida 32826
}

(Received 22 December 2006; accepted 28 February 2007; published online 2 April 2007)

\begin{abstract}
Dropping miscible and low-density organic solvents into water generates translational and rotational motions of floating objects including oil droplets, polymer half spheres, and model boats. The moving speed of the boat at different loads and the force produced by solvent drops are measured. In contrast to motions driven by surface tension of monolayer, the liquid mixing driven motion can be dynamically steered without restriction and continued provided the supply is maintained and the amount of water is large enough. Such motions are the result of Marangoni instability in binary liquid-liquid systems with intentionally produced concentration gradients behind the floating objects. (C) 2007 American Institute of Physics. [DOI: 10.1063/1.2719029]
\end{abstract}

The mixing of substances is an omnipresent phenomenon with fundamental importance. The characteristic increase of entropy during the mixing of two types of gaseous molecules at the same temperature and pressure is usually not associated with the generation of work. Although the mixing of two liquid substances is different due to the existence of clear surfaces, the process cannot generate exploitable works as well. Even if the liquids have different chemical potentials before mixing, the Gibbs free energy change in the spontaneous mixing process is dissipated as the random motions of molecules. The achievement of natural and manmade machines has attracted much attention in designing macroscopic devices capable of generating work from various energy sources. As an important form of energy, chemical energy can be exploited to generate work in biological systems or combustion engines. ${ }^{1}$ However, finding alternatives for energy exchange, overcoming the trend of randomness, and generating directional motions remain great challenges for the rational design and call for other mechanisms for energy management.

Camphor dancing and similar motions on the water surface have been known for several centuries, where the floating solid substances (camphor or surfactant) dissolve in water and form expanding monolayers on water. ${ }^{2,3}$ Due to the uneven shape of the solid piece, the dissolution of the solid occurs rapidly at a sharp point than that at a smooth surface. The concentration of the substance in water near the sharp point is greater than that at the other point, and the surface tension is least at the sharp point. Therefore the unbalanced surface tensions drive the motions on the water surface. However the surface tension driven motion lasts only for a limited time, because once a dense film of the molecule is formed on the water surface, there is no driving force to move the object. In addition, although there have been some practices intended to control the motion of the solid objects, the moving direction is determined by surface tension gradient. ${ }^{4}$ Thus the floating object cannot retake its previous trajectory. Here we demonstrate that the translational and rotational motions of floating objects can be driven by the dropwise mixing of water-miscible, low-density organic solvents into water. The motions can be sustained provided the

\footnotetext{
${ }^{a)}$ Electronic mail: mingsu@mail.ucf.edu
}

supply was maintained and the amount of water was sufficiently large. The moving direction can be dynamically steered by controlling the position of the droplet relative to the objects.

The liquid mixing driven motions can be achieved on both soft and hard floating objects. First a mineral oil droplet is placed on the water surface. An ionic surfactant (sodium laureth sulfate) is used to form liquid lens of oil on water by preventing the spreading of the oil droplet. The motion of the oil droplets is recorded continuously using a digital video recorder, and the consecutive images are obtained to derive the moving speed and analyze the shape change during the motion. Dropping an ethanol droplet next to a floating oil droplet deforms and pushes the oil droplet in the opposite direction at a speed of $\sim 6 \mathrm{~cm} / \mathrm{s}$; the oil droplet stops after traveling a few centimeters under the action of friction. By dropping ethanol at different directions, the motion of oil droplets can be steered to the desired direction. Two oil drops will move in opposite directions if an ethanol drop is deposited between them. When ethanol is dropped on the top of an oil droplet, the droplet expands on the water surface and immediately moves to a direction depending where the ethanol is leaking into water. The momentum transfer of the falling ethanol droplet is excluded, because dropping the same volume of water into water next to the oil droplet produces no such directional motion, except for some small motions induced by rippling.

Dropping ethanol next to polymeric objects floating on water produces similar motions. Hot-melt polyethylenepoly(vinyl acetate) (ACE glue stick) is used to make several half spheres by depositing melted polymer onto a cold glass substrate. After the polymer melt is cooled down to room temperature, the polymer half spheres can be detached from the glass and suspended on water without using a surfactant. The hydrophobic polymer has a water contact angle of $102.3^{\circ}$ and a density between those of water and ethanol. The weights of three half spheres are $0.15,0.052$, and $0.0068 \mathrm{~g}$. Dropping ethanol next to a floating half sphere $(0.052 \mathrm{~g})$ will move it to the opposite direction at a speed of $3.66 \mathrm{~cm} / \mathrm{s}$. The resistance preventing the continuous motion of the half sphere comes from the friction with water.

The liquid mixing driven motions can be realized in a controlled fashion. In the simplest format, a syringe tube 

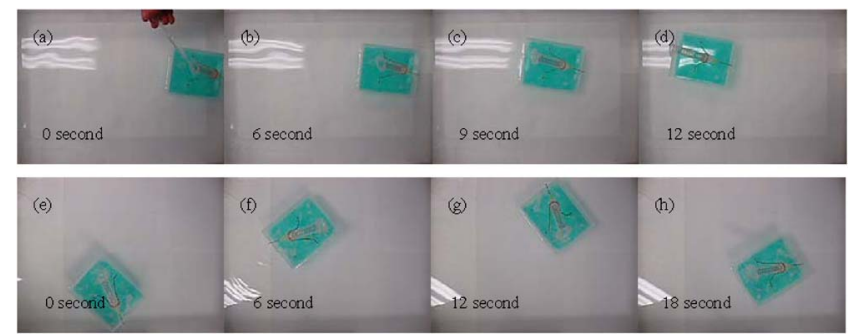

FIG. 1. (Color online) Dropping ethanol into water generates the motion of a plastic boat floating on water. By changing the relative position of ethanol outlet, the moving direction can be steered $[(\mathrm{a})-(\mathrm{d})]$ in a straight line, and $[(\mathrm{e})-(\mathrm{h})]$ in a circular track.

coupled with a medical injection needle is mounted on a plastic boat with a rectangular cross section. The syringe is tilted to an angle of $45^{\circ}$ and fixed on the boat that has a geometry of $13 \times 10.5 \times 3 \mathrm{~cm}^{3}$. The bottom of the boat is colored to enhance image contrast. The position of the syringe can be adjusted to change the direction of the driving force. A tank is filled with $50.5 \times 38 \times 4.5 \mathrm{~cm}^{3}$ water and used as a pool to float the boat. The boat will immerse $2 \mathrm{~mm}$ into water under its own weight $(39.4 \mathrm{~g})$, and the outlet of the injection needle is $23 \mathrm{~mm}$ above the water. For each experiment, the initial volume of ethanol is kept identical to avoid the difference in weight during motion. Ethanol flows via gravity and drops into the water at the same position relative to the boat. Extra weights are equally distributed on four corners of the boat. The speed is measured when the boat had moved in a straight line and averaged over three to five measurements. By continuously dropping ethanol from the syringe to the water, the boat moves smoothly across the water surface even when the weight of load is three times more than that of the boat [Figs. 1(a)-1(d)]. The direction of motion can be dynamically steered by dropping ethanol into water on one side of the boat. In the control experiment when pure water is dropped into the water, it takes an infinite amount of time for the boat to travel a distance of $36.5 \mathrm{~cm}$. In addition, by putting the injection needle on an off-centered position, the boat performs continuous circular motions once ethanol starts dropping into the water because of the generation of torsion from the unbalanced driving force [Fig. 1(e)-1(h)]. Such rotation motions can continue for several hours with no sign of stopping if the ethanol supply is sufficient.

The moving speed of the boat becomes smaller with increased load [Fig. 2(a)]; however, once the boat starts to move, the speed is constant. The force generated by ethanol is measured using the laser reflection method, where the boat is fixed on the free end of an elastic cantilever with the geometry of $60 \times 20 \times 0.25 \mathrm{~mm}^{3}$. The water level in the tank is adjusted to minimize the tilt of the floating boat. A small mirror is attached to the front side of the boat. A He-Ne laser beam is focused on the mirror and reflected into a quadrant position sensitive detector [Fig. 2(b)]. The signals are preamplified and processed using a self-made electronic module and recorded using an Agilent data acquisition unit. Each time, a $20 \mu \mathrm{l}$ ethanol is dropped in front of the boat and the bending of the cantilever is recorded. Dropping one drop of ethanol into water bends the cantilever, and the boat returns to its original position under the action of elastic energy stored in the bent cantilever, and dropping one drop of water into water produces a much smaller bending that is indistin-
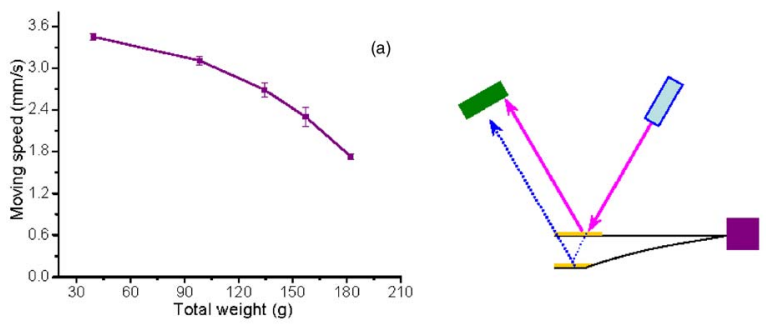

(b)
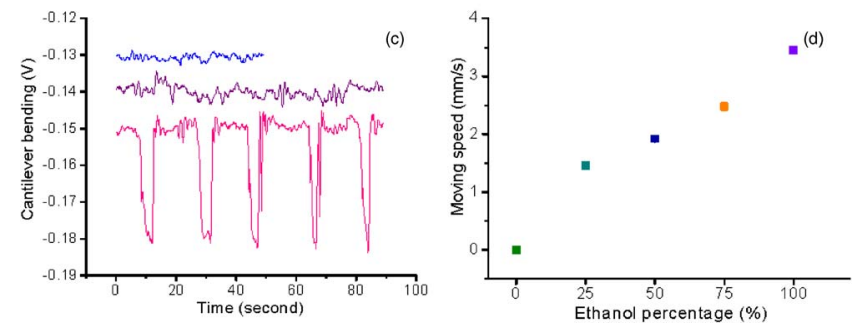

FIG. 2. (Color online) (a) Moving speed of the boat with different loads, where the starting volume of ethanol in syringe $(2 \mathrm{ml})$ is identical for each run, and the weight is equally distributed to avoid tilting. (b) Scheme of cantilever bending and force measurement. (c) Dropping five ethanol droplets (20 $\mu \mathrm{l}$ volume of each drop) into water bends the cantilever (red); dropping water in the same manner produces no significant motion (purple), which can hardly be distinguished from the background (blue). The curves are vertically shifted for comparison. (d) Dropping the mixtures of ethanolwater at a different volume ratio drives the motion of the boat.

guishable from the background [Fig. 2(c)]. The sensitivity of the position detection is $5.5 \times 10^{-4} \mathrm{~V} / \mu \mathrm{m}$, and the spring constant of the cantilever $(1.54 \mathrm{~N} / \mathrm{m})$ is determined from its bending at different loads. The force generated by one drop of ethanol $(162 \mathrm{mN})$ is derived with the assumption that the boat was not tilted at the small bending level. For a typical detection with a voltage change of $0.03 \mathrm{~V}$, the bending at the end of the cantilever $(105.2 \mu \mathrm{m})$ is derived from the shift of the laser spot in the detector $(54.5 \mu \mathrm{m})$ and the incident angle of the laser $\left(\sim 15^{\circ}\right)$. As a comparison, dropping one drop of water into water produces a much smaller force than that of the same volume of ethanol. In order to determine the effect of liquid dropping mode on the force, the force produced by the continuously dropped ethanol from the injection needle is measured $(624 \mu \mathrm{N})$ from the video image of a bending fiber (spring constant of $0.08 \mathrm{~N} / \mathrm{m}$, end displacement of $7.8 \mathrm{~mm}$ ). The duration of force in the single drop measurement is $\sim 5.4 \mathrm{~s}$, which corresponds to 12.6 drops in the continuous mode. Thus the force produced by $1 \mu \mathrm{l}$ ethanol in continuous mode is $7.3 \mu \mathrm{N}$, which is smaller than that in the single drop experiment $(8.1 \mu \mathrm{N})$, suggesting that the effective driving force per unit volume of ethanol is smaller in the continuous dropping mode.

At the constant temperature, the mixing of water and an organic solvent produces several changes such as the destruction of hydrogen network in water, the increase of entropy, and the change in viscosity. For a binary nonreactive liquid mixing system at ambient condition $\left(273{ }^{\circ} \mathrm{C}\right.$ and $1 \mathrm{~atm})$, the enthalpy change is zero, and the entropy of the mixing could be responsible for the motions. ${ }^{5}$ Experimentally, we found that dropping an ethanol-water mixture with a different ratio into water or ethanol in the 50:50 $(v / v)$ ethanol-water mixture produces similar motions. However, as expected, the moving speed of the boat driven by waterdiluted ethanol is slower than that of pure ethanol [Fig. 2(d)], confirming the smaller driving force even if other conditions are identical. Dropping water into ethanol or into the 50:50 

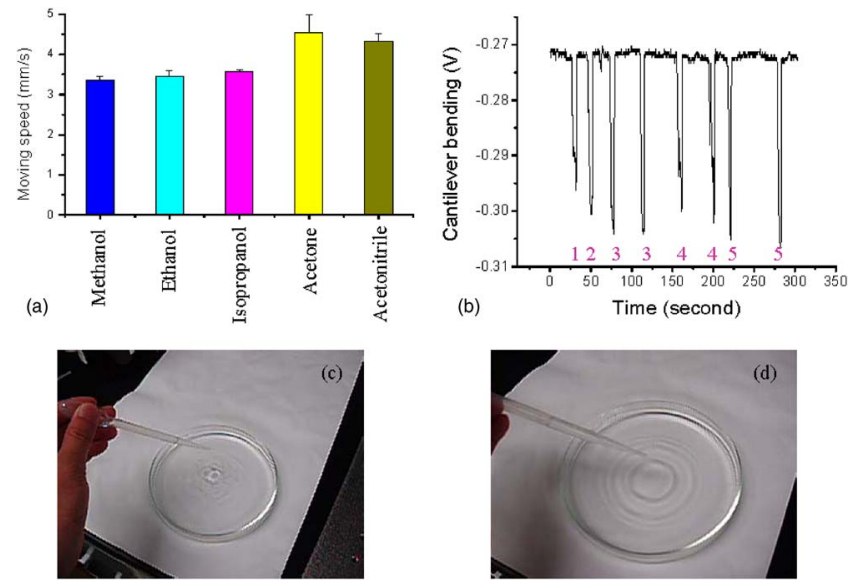

FIG. 3. (Color online) (a) Moving speed of an unloaded boat driven by different solvents. (b) Bending of a cantilever induced by $20 \mu \mathrm{l}$ of each solvent: the numbers from 1 to 5 stand for methanol, ethanol, isopropanol, acetone, and acetonitrile, respectively. (c) Dropping ethanol into water. (d) Dropping water into water.

$(v / v)$ ethanol-water mixture does not move floating objects. By using colored water, we found that water will sink quickly in ethanol (1 cm deep), spread on the container floor, and shake a polymer half sphere sitting there.

Such motion is not limited to ethanol; other low-density, water-miscible organic solvents such as methanol, isopropanol, acetone, and acetonitrile will drive the boat with similar speed and force. The moving speed of the boat is measured when different solvents are used [Fig. 3(a)]. To avoid interference between solvents, water in the tank is changed after each solvent. Although the starting volumes are identical, the flow rate and the drop size of each solvent vary due to the difference in the cohesion energy of the solvent and the surface tension on the injection needle. The drop rate, flow rate, density, and drop size have been measured for each solvent, and the large drop rates are measured using video imaging. Even the flow rate of the solvent cannot be controlled precisely in the current design; the moving speeds of the boat are close to each other for the studied solvents. In addition, we have measured the force generated by $20 \mu \mathrm{l}$ of each solvent using the cantilever [Fig. 3(b)], where all solvents studied here show the same order of force.

High-speed imaging is then used to record the morphologic changes that occurred during the mixing of ethanol and water: ethanol drops generate characteristic turbulent mixing patterns with small and random fluctuations on the water [Fig. 3(c)]. In contrast, the water drops produce remarkable ripples [Fig. 3(d)]. It is known that ethanol can reduce the surface tension of water, as demonstrated in "tears of wine," has a lower density than water, and a strong tendency to form a macroscopically homogeneous mixture with water. Recent $\mathrm{x}$-ray absorption spectroscopy shows that ethanol mixes slightly with water at microscopic level, suggesting its hydrophobic nature. ${ }^{7}$ The interfacial turbulence in the mixing of ethanol and water has been proposed as the result of concentration gradient induced Marangoni instability. ${ }^{8}$ Principally dropping ethanol into water produces a localized gradient of ethanol concentration, which generates a surface tension that can drive the floating objects. The dropped ethanol dissolves into water immediately and forms a mixture. Since the concentration difference between ethanol and the mixture always exists, the mixing driven motion should be continued if ethanol supply is maintained. The translational motions of objects driven by chemical energy have been studied extensively in the past. ${ }^{9-12}$ However, most of previous researches are based on liquid droplets, which sit on self-assembled monolayer modified solid substrates. The method described here is based on a different mechanism and has the potential to become a generalized approach to convert chemical energy into work.

${ }^{1}$ G. Bottari, F. Dehez, D. A. Leigh, P. J. Nash, E. M. Pérez, J. K. Y. Wong, and F. Zerbetto, Angew. Chem., Int. Ed. Engl. 115, 6066 (2003).

${ }^{2}$ S. Nakata and Y. Hayashima, J. Chem. Soc., Faraday Trans. 94, 3655 (1998).

${ }^{3}$ S. Bekki, M. Vignes-Adler, E. Nakache, and P. M. Adler, J. Colloid Interface Sci. 492, 506 (1990).

${ }^{4}$ S. Ross and P. Becher, J. Colloid Interface Sci. 149, 575 (1992).

${ }^{5}$ I. Tinoco, K. Sauer, and J. C. Wang, Physical Chemistry: Principles and Applications in Biological Sciences (Prentice-Hall, Englewood Cliffs, NJ, 1985), Vol. 2, p. 612.

${ }^{6}$ A. E. Hosoi and J. W. M. Bush, J. Fluid Mech. 442, 217 (2001).

${ }^{7}$ J.-H. Guo, Y. Luo, A. Augustsson, S. Kashtanov, J.-E. Rubensson, D. K. Shuh, H. Agren, and J. Nordgren, Phys. Rev. Lett. 91, 157401 (2003).

${ }^{8}$ S. Slavtchev and M. A. Mendes, Int. J. Heat Mass Transfer 47, 3269 (2004).

${ }^{9}$ M. K. Chaudhury and G. M. Whitesides, Science 12, 1539 (1992).

${ }^{10}$ K. Ichimura, S.-K. Oh, and M. Nakagawa, Science 288, 1624 (2000).

${ }^{11}$ J. Berna, D. A. Leigh, M. Lubomska, S. M. Mendoza, E. M. Perez, P. Rudolf, G. Teobaldi, and F. Zerbetto, Nat. Mater. 4, 704 (2005).

${ }^{12}$ S.-W. Lee, D. Y. Kwok, and P. E. Laibinis, Phys. Rev. E 65, 051602 (2002). 